\title{
Mitigating dietary aflatoxins in Wistar rat using selected phyto-antioxidant sources
}

${ }^{1}$ Ewuola, E. O. and ${ }^{2 *}$ Emerue, P. C.

${ }^{\prime}$ Animal Physiology and Bioclimatology, Department of Animal Science, University of Ibadan

${ }^{2}$ Institute of Agricultural Research and Training, Moor Plantation, Ibadan. Corresponding author: patolala77@gmail.com; +2349012042609

\section{Abstract}

This study was conducted to investigate the mitigation of dietary aflatoxin in Wistar rats using extract from selected phyto-antioxidant sources. A total of one hundred and twenty Wistar rats weighing between 180-190g (80 females and 40 males) at six weeks old were allotted to eight treatments with 15 rats per treatment (10 females and 5 males), in a completely randomized design. The treatments were Treatment 1 (Normal diet + no extract and no aflatoxin), Treatment 2 (Aflatoxin contaminated diet + no extract), Treatment 3 (Aflatoxin contaminated diet $+100 \mathrm{mg} / \mathrm{kg} \mathrm{BW}$ Carrot extract), Treatment 4 (Aflatoxin contaminated diet $+200 \mathrm{mg} / \mathrm{kg} \mathrm{BW}$ Carrot extract), Treatment 5 (Aflatoxin contaminated diet $+100 \mathrm{mg} / \mathrm{kg} \mathrm{BW}$ Ginger extract), Treatment 6 (Aflatoxin contaminated diet $+200 \mathrm{mg} / \mathrm{kg} \mathrm{BW}$ Ginger extract), Treatment 7 (Aflatoxin contaminated diet $+100 \mathrm{mg} / \mathrm{kg} B W$ Garlic extract), Treatment 8 (Aflatoxin contaminated diet $+200 \mathrm{mg} / \mathrm{kg} \mathrm{BW} \mathrm{Garlic} \mathrm{extract).} \mathrm{Body} \mathrm{weight} \mathrm{gain}$ and feed conversion ratio of Wister rats (T8) administered $200 \mathrm{mg} / \mathrm{kg} \mathrm{BW}$ was significant $(p<0.05)$, with higher weight gain in male (206.00g) and female (199.70g). However, eviscerated and organs weights of both male and female Wistar rats was similar $(p>0.05)$ across the treatments. Haematological and serum biochemical indices among the treatments was not significant $(p>0.05)$, except for the globulin in male Wistar rats that differed significantly $(p<0.05)$ with the value $(5.00 \mathrm{~g} / \mathrm{dL})$ being higher in $T 7$. In conclusion, $200 \mathrm{mg} / \mathrm{kg}$ body weight of garlic extract improved growth rate of Wistar rats, without any deleterious effect on haematological and serum biochemical parameters. Therefore, $200 \mathrm{mg} / \mathrm{kg}$ body weight garlic extract mitigated the adverse effect of aflatoxin contaminated feed in Wistar rats.

Keywords: Wistar rat, phyto-antioxidants, aflatoxin, plant extract.

Atténuer les aflatoxines alimentaires chez le rat 'Wistar' à l'aide de certaines sources phyto-antioxydantes

\section{Résumé}

Cette étude a été menée pour étudier l'atténuation de l'aflatoxine diététique chez les rats de 'Wistar' tout en employant l'extrait des sources phyto-antioxydantes choisies. Un total de cent vingt rats Wistar pesant entre 180 et $190 \mathrm{~g}$ (80 femelles et 40 mâles) à l'âge de six semaines ont été attribués à huit traitements avec 15 rats par traitement (10 femelles et 5 mâles), dans une conception complètement randomisée. Les traitements étaient le traitement 1 (régime normal + aucun extrait et aucune aflatoxine), traitement 2 (régime contaminé par l'aflatoxine + aucun extrait), traitement 3 (régime contaminé par l'aflatoxine + extrait de carotte $B W$ de $100 \mathrm{mg} / \mathrm{kg}$ ), traitement 4 (régime contaminé par l'aflatoxine + extrait de carotte $B W$ de $200 \mathrm{mg} / \mathrm{kg}$ ), traitement 5 (Aflatox alimentation contaminée par l'aflatoxine + extrait de gingembre $B W 100 \mathrm{mg} / \mathrm{kg}$ ), Traitement 6 (régime contaminé à l'aflatoxine + extrait de gingembre $B W 200 \mathrm{mg} / \mathrm{kg}$ ), Traitement 7 (régime contaminé par l'aflatoxine $+100 \mathrm{mg} / \mathrm{kg}$ extrait d'ail BW), Traitement 8 (régime contaminé à l'aflatoxine $+200 \mathrm{mg} / \mathrm{kg}$ extrait d'ail 


\section{Mitigating dietary aflatoxins in Wistar rat using selected phyto-antioxidant sources}

$B W)$. Le gain de poids corporel et le rapport de conversion des aliments pour animaux des rats wisters (T8) administrés $200 \mathrm{mg} / \mathrm{kg} \mathrm{BW}$ étaient significatifs $(p<0,05)$, avec un gain de poids plus élevé chez les rats Wistar mâles (206,00 g) et femelles (199,70 g). Cependant, les poids éviscérés et organes des rats Wistar mâles et femelles étaient similaires $(p>0,05)$ à travers les traitements. Les indices biochimiques hématologiques et sériques parmi les traitements n'étaient pas significatifs $(p>0,05)$, à l'exception de la globuline chez les rats wistar mâles qui différait considérablement $(p<0,05)$ avec la valeur $(5,00 \mathrm{~g} / \mathrm{dL})$ étant plus élevée dans T7. En conclusion, le poids corporel de $200 \mathrm{mg} / \mathrm{kg}$ de l'extrait d'ail a amélioré le taux de croissance des rats de Wistar, sans n'importe quel effet délétère sur les paramètres biochimiques hématologiques et sériques. Par conséquent, l'extrait d'ail de poids corporel de $200 \mathrm{mg} / \mathrm{kg}$ a atténué l'effet défavorable de l'alimentation contaminée d'aflatoxin chez les rats de Wistar.

Mots-clés : Rat Wistar, phyto-antioxydants, aflatoxine, extrait de plante.

\section{Introduction}

Globally, aflatoxin has been the major toxic substance affecting adequate production of animal protein. Adverse effects of aflatoxin on animal health and production amongst others include liver damage, gastrointestinal dysfunction, decreased feed utilization and efficiency, decrease in egg production, poor egg-shell quality in poultry and inferior carcass quality (Devegowda et al., 1998; Charoenpornsook and Kavisarai, 2006). Consumption of high levels aflatoxin contaminated cereals and oilseeds diets have been implicated in reduction of growth and overall health of animals. Four major aflatoxins produced in feedstuffs are Aflatoxin B1, Aflatoxin B2, Aflatoxin G1 and Aflatoxin G2. Research also indicated that only four species of fungi produce aflatoxins namely, Aspergillus parasiticus, Aspergillus nominus, Aspergillus pseudotamarii, Aspergillus flavus (Kurtzman et al., 1987, Payne, 1998; Ito et al., 2001; Cortyl, 2008). However, only Aspergillus flavus and Aspergillus parasiticus are of economic importance. Since animal feedstuffs are major input needed for feed formulation to meet the nutritional requirements of livestock, contamination by aflatoxins could affect growth and performance. Hence, the approach of mitigating the adverse effect of aflatoxin contamination of feed is paramount. Several approaches of mitigating aflatoxin contamination in food and feed such as the use of microbes and enzymes capable of degrading tricothecene have been documented (Wilson et al., 2017). In addition, Gomez-Espinosa et al. (2017) reported the use of neutral electrolyzed water to prevent aflatoxicosis in turkey poults. However, none of these approaches has been able to mitigate dietary contamination of feed by aflatoxin using Wistar rats as an experimental animal.

Phytotheraphy has been the back bone of medicine in which various herbs and their extract containing active ingredient of therapeutic significance are used. Therefore, it is necessary to understand the effects of phytochemical feed additives in terms of cost effectiveness and comparatively less side effect (Mahima et al., 2012, 2013) when compared with antibiotic feed additive. The consumption of phytochemical rich foods such as fruits, vegetables, spice are associated with a reduced risk of diseases mediated by oxidative stress and inflammation such as certain cancers, atherosclerosis and neurodegenerative diseases Halliwell, (1994). Several plants and their constituents are rich source of antioxidant and play a significant role in prevention of disease progression process thus qualifying the leaves as a potential animal feed supplement (Moyo et al., 2011). However, there are several naturally existing phyto- 


\section{Ewuola and Emerue}

antioxidant sources, especially ginger, carrot, and garlic in which studies have shown some effective potential. Garlic (Allium sativum) is the most important specie of the onion genus, Allium belonging to the family Alliaceae (Eric, 2010). Intact garlic bulbs contain alliin (S-allyl cystein sulfoxide), the precursor of allicin (S-allyl2propenthiosulphate), which is hydrolyzed by enzyme allinase upon crushing to its active form, and the allicin (S-allyl2 propenthiosulphate) has the agent responsible for garlic's potent antibacterial properties. Garlic has been shown to increase feed palatability and thus feed intake (Horton et al., 1991). Allicin is the most potentially active component of garlic that is responsible for its characteristic odour, flavor as well as most of its biological properties (Chowdhury et al., 2002). Tollba and Hassan (2003) found that garlic as a natural feed additive, improved broilers growth, feed conversion ratio (FCR) and decreased mortality rate. The whole use of plant (bulb) and plant extract singly have been documented with positive effect. Ginger (Zingiber officinale), on the other hand, is a rhizomatous herbaceous perennial herb, and a member of the Zingiberaceae family. The rhizome of ginger (Zingiber officinale) are considered to have natural medicinal properties, including anti-inflammatory (Ammon et al 1993), antioxidant, anticarcinogenic, antimutagenic, anticoagulant, antidiabetic, antibacterial, antifungal, antiprotozoal (Antony et al., 1999), antiviral, antifibrotic, antivenom, anti-ulcer, hypotensive, and hypocholesterolemic (Al-Yahya et al., 1989), anti-hypertensive (vasolidator), antithrombotic, hypoglycaemic, hypolipidemic, and anti-pyretic properties because they contains a number of monoterpenoids, sesquiterpinoids, curcuminoids, sesquiterpene-Zingiberene, and pungent phenol compounds such as gingerols and shagoals (Soni et al., 1997).
Different researchers have worked on the effect of ginger on growth performance in broilers, and controversial results were reported. Taylor (2001) observed that the use of ginger powders significantly increased body weight and improved feed conversion compared to birds fed with control diet. In contrast, Zhang et al (2009) examined the effect of processed ginger on growth performance and showed that ginger additive had no significant effect on the feed efficiency, while body weight gain of birds fed ginger supplement were higher than control groups. Carrot (Daucus carota) is one of the popular root vegetables, the presence of high concentration of antioxidant carotenoids especially $\beta$ carotene may account for the biological and medicinal properties of carrots. Carrots have been reported to have diuretic, $\mathrm{N}$ balancing properties and are effective in the elimination of uric acid (Anon, 1952). Numerous animal experiments and epidemiological studies have indicated that carotenoids inhibit carcinogenesis in mice and rats and may have anti-carcinogenic effects in humans. In biological systems, $\beta$ carotene functions as a free radical-trapping agent and single oxygen quencher and have antimutagenic, chemopreventive, photoprotective and immunoenhancing properties (Deshpande et al., 1995). Studies have shown that the presence of $\alpha$ - and $\beta$ carotene in blood has a protective effect against atherosclerosis (D'Odorico et al., 2000). However, the use of phytoantioxidant to mitigate dietary aflatoxin has not received much attention. Hence, their use in aflatoxin contaminated diet for Wistar rat requires investigation. This research was therefore designed to assess the efficiency of the extract of Ginger (Zingiber officinale), Garlic (Allium sativum), and Carrot (Daucus carota) in mitigating dietary aflatoxin using Wistar rat as a model. 
Mitigating dietary aflatoxins in Wistar rat using selected phyto-antioxidant sources

Materials and methods

Experimental site and processing of test samples

The experiment was conducted at the Rat House of the Department of Animal Science, University of Ibadan, Nigeria. Fresh samples of Garlic, ginger and carrot were purchased from Bodija market in Ibadan. Part of the fresh samples were first subjected to phytochemical analysis to quantify the tannins, saponins, flavonoids, alkaloids, terpenoids, carotenoids, and antioxidant using ORAC (Oxygen Radical Absorbance Capacity).

\section{Preparation of extract}

Fresh samples of ginger, garlic and carrot were sliced into surface area, oven dried and milled into powder. Carrot was first soaked and rinsed in salt solution of $20 \mathrm{~g}$ in 2litres of water $(2 \% \mathrm{NaCl}$ solution) to maintain its colour because of heat. The extraction was carried out using ethanol as described (Mohan, 2004). A liter of $80 \%$ ethanol solution was mixed with $200 \mathrm{~g}$ of powdered ginger, garlic and carrot, respectively. The mixtures were kept for 5 days in tightly sealed vessels at room temperature of $22^{\circ} \mathrm{C}$ and kept away from sunlight. The mixtures were stirred several times daily with a sterile glass rod. At the fifth day, each mixture was filtered using muslin cloth and the residue adjusted to the required concentration $(500 \mathrm{ml}$ of $80 \%$ ethanol for the residue of $200 \mathrm{~g}$ each of the ginger, garlic and carrot powdered materials) with the ethanol for further extraction. Extraction of the residue was repeated 3-5 times until a clear colourless supernatant liquid was obtained indicating that no more extraction from the plant material was possible. Thereafter, a 2-liter aliquot of extracted liquid each was subjected to rotary evaporation for 1-2 hours using Brinkmann Rotavapor Model to remove the ethanol. A known quantity $(5 \mathrm{mls})$ of the semi-solid extract produced was subjected to antioxidant analysis and later kept in a deep freezer at a temperature of $-80^{\circ} \mathrm{C}$ until further use.

\section{Experimental animals and management}

One hundred and twenty (120) Wistar rats with body weight (BW) of 180-190g at six weeks old were used for the experiment ( 80 females and 40 males) in a 70 day feeding trial. The Wister rats were allotted into eight treatments groups of 15 rats (10 females and 5 males) in a completely randomized design. Animals were acclimatized to individual cubicles for at least three weeks before the start of the experiment. The female rats were replicated into 2 ( 5 rats per replicate) and 5 male rats in each cubicle. The dietary treatments as indicated below were fed once daily to the rats according to their body weight. Clean and cool water was offered ad libitum. Rats were administered extracts orally three times in a week according to their BW as highlighted below using cannula. Weight gain were determined weekly before early morning feeding. Feed intake was obtained by subtracting the leftover feed from the quantity served. Weight gain of the rats was determined by subtracting the initial weight from the final weight gain. Feed conversion ratio was obtained by dividing the average daily feed intake by the average body weight gain.

\section{Experimental diet}

$\mathrm{T} 1=$ Normal diet + No extract and no aflatoxin

$\mathrm{T} 2=$ Aflatoxin contaminated diet + No extract

T3= Aflatoxin contaminated diet $+100 \mathrm{mg} / \mathrm{kg} \mathrm{BW}$ Carrot Extract

T4= Aflatoxin contaminated diet + 200mg/kg BW Carrot Extract

$\mathrm{T}=$ 5Aflatoxin contaminated diet + $100 \mathrm{mg} / \mathrm{kg} \mathrm{BW}$ Ginger Extract

$\mathrm{T} 6=$ Aflatoxin contaminated diet + $200 \mathrm{mg} / \mathrm{kg}$ BW Ginger Extract

$\mathrm{T} 7=$ Aflatoxin contaminated diet + $100 \mathrm{mg} / \mathrm{kg} \mathrm{BW}$ Garlic Extract

$\mathrm{T} 8=$ Aflatoxin contaminated diet + $200 \mathrm{mg} / \mathrm{kg}$ BW Garlic Extract 
Ewuola and Emerue

Table 1: Ingredient composition of experimental diets (\%)

\begin{tabular}{llll}
\hline Control group & & Aflatoxin contaminated & Diet \\
\hline Ingredients & Quantity (kg) & Ingredients & Quantity (kg) \\
\hline Maize & 40.00 & Maize & 35.20 \\
----- & ----- & Contaminated maize & 4.80 \\
Soya bean & 40.00 & Soya bean & 40.00 \\
Wheat offal & 13.15 & Wheat offal & 13.15 \\
Di-calcium phosphate & 2.50 & Di-calcium phosphate & 2.50 \\
Limestone & 2.50 & Limestone & 2.50 \\
Salt & 0.65 & Salt & 0.65 \\
Methionine & 0.60 & Methionine & 0.60 \\
Lysine & 0.60 & Lysine & 0.60 \\
Total & $\mathbf{1 0 0 . 0 0}$ & Total & $\mathbf{1 0 0 . 0 0}$ \\
Determined analysis & & Determined analysis & \\
Energy(Kcal/kg ME) & 2600 & Energy (Kcal/kg ME) & 2600 \\
Crude protein (\%) & 20 & Crude protein (\%) & 20 \\
----- & ---- & Aflatoxin (ppb) & 200 \\
\hline
\end{tabular}

$\mathrm{ppb}=$ part per billion

\section{Blood collection and evaluations}

At the end of feeding trial, three rats from each replicate of female and male were bled individually from the retro-orbital venous plexus according to the standard procedure for haematology and serum biochemical analysis (Girling et al, 2015). Blood samples were collected into separate sterilized glass tubes containing EDTA (ethylene-diamine-tetra-acetic acid) and another glass tube without anticoagulant for haematological and serum biochemical assay, respectively. Blood samples for serum were centrifuged and serum was decanted and freeze stored until analysis. Blood samples collected were analyzed for Packed cell volume, haemoglobin, red blood cell (RBC), white blood cell (WBC), platelets, lymphocyte, neutrophils, monocytes and eosinophils as outlined in Ewuola and Egbunike, (2008). The blood samples collected for serum biochemical analysis were centrifuged at 3500 revolutions per minute (rpm) for 15 mins. The serum was collected and analyzed for the following parameters; aspartate aminotransferase (AST), alanine aminotransferase (ALT), and alkaline-phosphate (ALP), activities were determined using spectrophotometric methods (McComb et al, 1988), Serum total protein was determined by Biuret method (Kohn and Allen, 1995), while albumin was determined using the BCG (Bromocresol green) method (Peter et al, 1982), Serum urea was determine by Urease method and creatinine by Foin-wu filtrate methods (Toro and Ackermann, 1975).

\section{Statistical analysis}

Data collected were subjected to ANOVA according to the procedure of SAS (1999) and means were separated using Duncan's Multiple Range Test (Steel and Torrie, 1990).

\section{Results \\ Growth indices of the Wistar rats administered extract from selected phyto- antioxidant sources}

Growth indices of the male Wistar rats fed with selected phyto-antioxidant sources is presented in Table 2. There were significant differences $(p<0.05)$ in the values obtained for the total feed intake and feed conversion ratio across the treatment groups. The rats in $\mathrm{T} 4$ had the highest feed conversion ratio of 5.15, while those in T8 had the least (4.28). Results of the body weight gain was 


\section{Mitigating dietary aflatoxins in Wistar rat using selected phyto-antioxidant sources}

significantly $(\mathrm{p}<0.05)$ higher in the Wistar rats fed T8 $(206.00 \mathrm{~g})$ than others while those fed T2 had the least $(193.95 \mathrm{~g})$ body weight gain. Growth indices of the female wistar rats administered extract from selected phyto-antioxidant source is presented in Table 3. There was no significant difference in the value obtained for the total feed intake. The body weight gain showed significant difference $(\mathrm{p}<0.05)$ with T8, T3, T4, T5 and T6 having higher significant values of $199.70 \mathrm{~g}, 196.10 \mathrm{~g}$, $197.10 \mathrm{~g}, 196.80 \mathrm{~g}$ and $198.70 \mathrm{~g}$, respectively than T1 (153.25) and T2 (151.70). The feed conversion ratio was significantly different $(p<0.05)$ across the treatment groups with rats administered $\mathrm{T} 4$ having higher significant value (4.61) than other treatment groups, while the T8 rats had the least (3.98) feed conversion ratio.

The eviscerated and organ weight of the Wistar rats administered extract from selected phyto-antioxidant sources

Relative eviscerated and internal organs weight of the Wistar rats administered extract from selected phyto-antioxidant sources is shown in Table 4 and 5. There was no significant difference in the eviscerated weight of the Wistar rats and their internal organs among the treatment groups.

Table 2: Growth indices of male Wistar rats administered extract from selected phyto-antioxidant sources

\begin{tabular}{|c|c|c|c|c|c|c|c|c|c|}
\hline Parameters & $\mathrm{T} 1$ & $\mathrm{~T} 2$ & $\mathrm{~T} 3$ & $\mathrm{~T} 4$ & $\mathrm{~T} 5$ & T6 & $\mathrm{T} 7$ & T8 & $\begin{array}{l}\text { P- } \\
\text { value }\end{array}$ \\
\hline TFI (g/day) & $39.50 \pm 2.25^{\mathrm{a}}$ & $34.00 \pm 0.45^{b}$ & $36.00 \pm 5.75^{\mathrm{ab}}$ & $35.50 \pm 5.75^{\mathrm{a}}$ & $38.50 \pm 2.65^{\mathrm{a}}$ & $37.00 \pm 5.90^{\mathrm{ab}}$ & $37.00 \pm 2.75^{\mathrm{ab}}$ & $38.50 \pm 160^{\mathrm{ab}}$ & 0.12 \\
\hline $\begin{array}{l}\text { IBW } \\
\text { (g/day) }\end{array}$ & $183.50 \pm 1.50$ & $183.10 \pm 2.30$ & $182.75 \pm 0.55$ & $185.20 \pm 2.80$ & $186.75 \pm 1.05$ & $183.60 \pm 1.40$ & $183.80 \pm 1.20$ & $186.50 \pm 0.90$ & 0.54 \\
\hline $\begin{array}{l}\text { BWG } \\
\text { (g/day) }\end{array}$ & $203.20 \pm 2.80$ & $193.95 \pm 0.05$ & $200.75 \pm 1.05$ & $198.80 \pm 0.80$ & $200.70 \pm 0.00$ & $199.25 \pm 0.75$ & $200.80 \pm 0.30$ & $206.00 \pm 0.60$ & 0.25 \\
\hline FCR & $4.71 \pm 0.45^{\mathrm{ab}}$ & $4.85 \pm 0.02^{\mathrm{c}}$ & $4.54 \pm 0.03^{b c}$ & $5.15 \pm 0.09^{\mathrm{a}}$ & $5.08 \pm 0.09^{\mathrm{ab}}$ & $4.96 \pm 0.07^{\mathrm{ab}}$ & $4.97 \pm 0.04^{\mathrm{ab}}$ & $4.28 \pm 0.04^{\mathrm{ab}}$ & 0.07 \\
\hline $\begin{array}{l}\text { abc }=\text { Means } \\
\text { TFI= Total } \mathrm{f} \\
\text { Tl= No Afla } \\
\text { BW Carrot } \\
\text { weight ging } \\
\text { body weight }\end{array}$ & $\begin{array}{l}\text { on the same rov } \\
\text { feed intake; IBV } \\
\text { atoxin and extr } \\
\text { Extract; T4 = A } \\
\text { er extract; T6= } \\
\text { t garlic extract; }\end{array}$ & $\begin{array}{l}W \text { with different } \\
N=\text { Initial body } \\
\text { ract (control); T } \\
\text { flatoxin }+200 \\
\text { Aflatoxin }+20 \\
T 8=\text { Aflatoxin }\end{array}$ & $\begin{array}{l}\text { t superscripts ar } \\
\text { weight; BWG= } \\
\text { [2 = Diet + Afla } \\
\mathrm{mg} / \mathrm{kg} \mathrm{BW} \mathrm{Car} \\
0 \mathrm{mg} / \mathrm{kg} \text { body w } \\
+200 \mathrm{mg} / \mathrm{kg} \text { bo }\end{array}$ & $\begin{array}{l}\text { e significantly } \\
=\text { Body weigh } \\
\text { toxin + No ex } \\
\text { rot Extract l; } \\
\text { reight ginger } \\
\text { dy weight gar }\end{array}$ & $\begin{array}{l}(\mathrm{P}<0.05) \text { diff } \\
\text { gain; FCR= Fe } \\
\text { ract; T3= Afla } \\
5=\text { Aflatoxin } \\
\text { xtract; } \mathrm{T} 7=\mathrm{Af} \\
\text { ic extract }\end{array}$ & $\begin{array}{l}\text { rent. } \\
\text { d conversion ra } \\
\text { oxin }+100 \mathrm{mg} \\
+100 \mathrm{mg} / \mathrm{kg} \text { bo } \\
\text { atoxin }+100 \mathrm{mg}\end{array}$ & & & \\
\hline
\end{tabular}

Table 3: Growth indices of female Wistar rats administered extract from selected phyto-antioxidant sources

\begin{tabular}{llllllllll}
\hline Parameter & T1 & T2 & T3 & T4 & T5 & T6 & T7 & T8 & P-value \\
\hline TFI(g/day) & $39.50 \pm 0.50^{\mathrm{a}}$ & $34.00 \pm 1.00^{\mathrm{c}}$ & $36.50 \pm 1.50^{\mathrm{abc}}$ & $35.00 \pm 1.00^{\mathrm{bc}}$ & $38.00 \pm 1.00^{\mathrm{ab}}$ & $37.00 \pm 1.00^{\mathrm{abc}}$ & $37.00 \pm 1.00^{\mathrm{abc}}$ & $38.50 \pm 1.50^{\mathrm{ab}}$ & 0.09 \\
$\mathrm{IBW}(\mathrm{g} /$ day $)$ & $180.10 \pm 1.05$ & $180.00 \pm 0.55$ & $185.20 \pm 2.80$ & $184.30 \pm 1.20$ & $186.20 \pm 1.05$ & $187.15 \pm 0.90$ & $181.35 \pm 0.55$ & $186.30 \pm 1.40$ & 0.46 \\
BWG(g/day) & $183.25 \pm 3.05^{\mathrm{b}}$ & $181.70 \pm 1.70^{\mathrm{b}}$ & $196.10 \pm 1.10^{\mathrm{a}}$ & $197.10 \pm 1.10^{\mathrm{a}}$ & $196.80 \pm 1.80^{\mathrm{a}}$ & $198.70 \pm 1.70^{\mathrm{a}}$ & $185.55 \pm 2.05^{\mathrm{ab}}$ & $199.20 \pm 0.20^{\mathrm{a}}$ & 0.01 \\
FCR & $4.06 \pm 0.05^{\mathrm{c}}$ & $4.46 \pm 0.08^{\mathrm{ab}}$ & $4.39 \pm 0.15^{\mathrm{ab}}$ & $4.61 \pm 0.10^{\mathrm{a}}$ & $4.06 \pm 0.06^{\mathrm{bc}}$ & $4.23 \pm 0.07^{\mathrm{ab}}$ & $4.26 \pm 0.06^{\mathrm{bc}}$ & $3.98 \pm 0.08^{\mathrm{c}}$ & 0.01 \\
\hline
\end{tabular}

abc= Means on the same row with difference superscripts are significantly different $(\mathrm{P}<0.05)$

TFI= total feed intake; $\mathrm{BWG}=$ Body weight gain; FCR = feed conve rsion ratio; T1= No Aflatoxin and extract (control); T2= Aflatoxin + No extract, T3=Aflatoxin $+100 \mathrm{mg} / \mathrm{kg}$ BW carrot extract; T4 Aflatoxin $+200 \mathrm{mg} / \mathrm{kg}$ BW carrot extract; T5= Aflatoxin + 100mg $/ \mathrm{kg}$ BW ginger extract; T6= Aflatoxin + 200mg $/ \mathrm{kg}$ BW ginger extract; T7= Aflatoxin $+100 \mathrm{mg} / \mathrm{kg}$ BW garlic extract; T8= Aflatoxin $+200 \mathrm{mg} / \mathrm{kg}$ BW garlic extract. 


\section{Ewuola and Emerue}

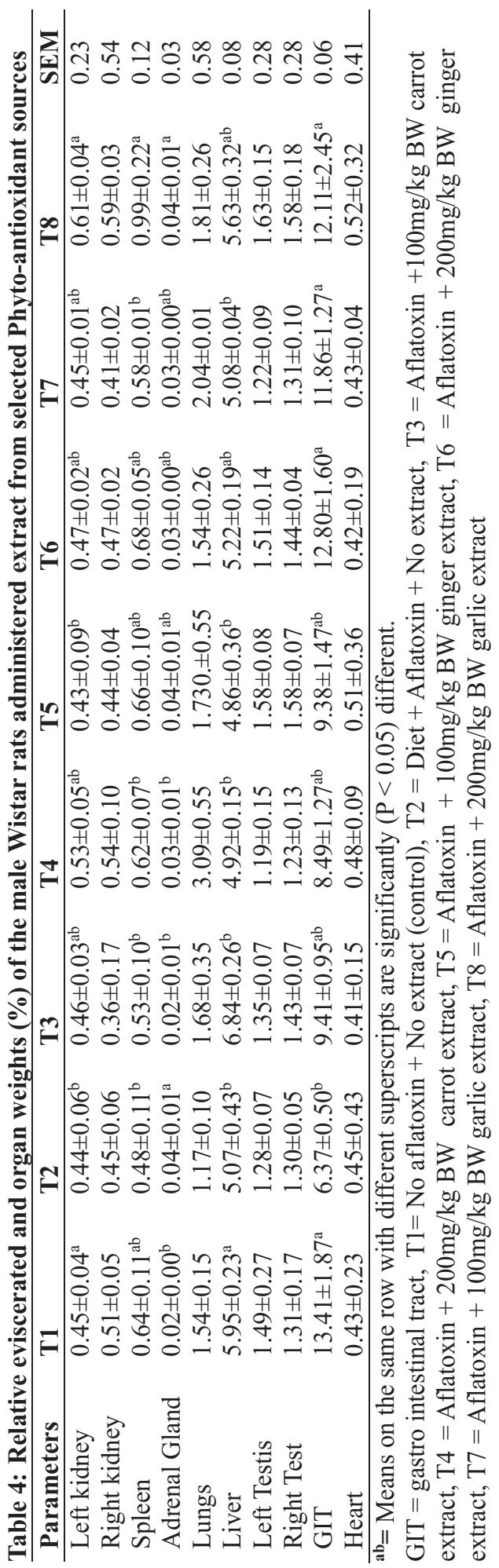




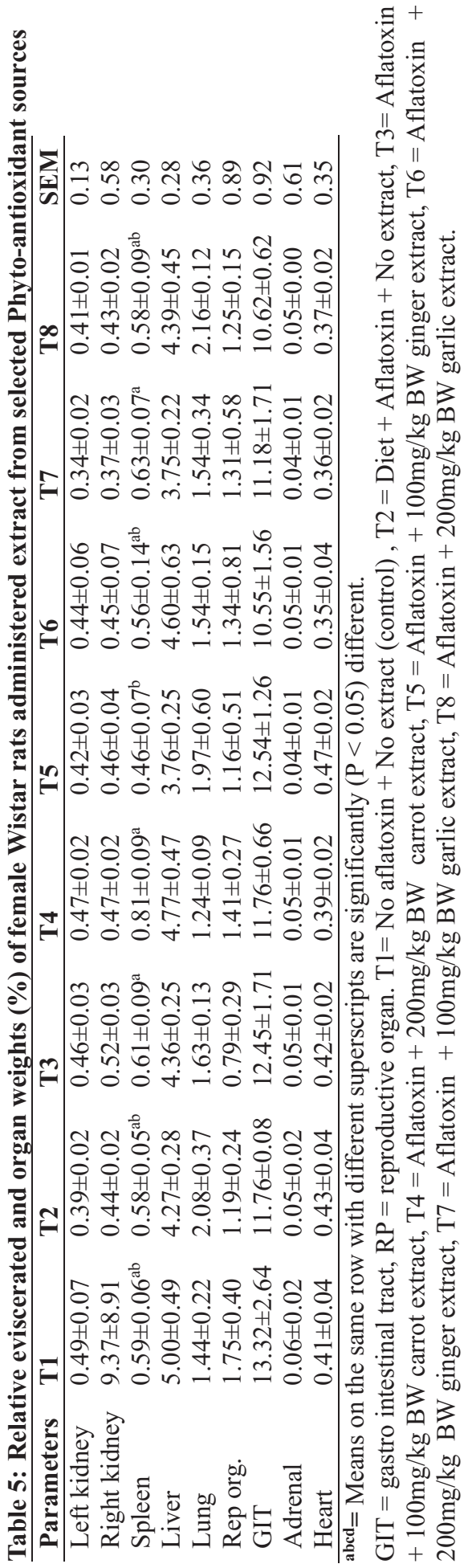




\section{Ewuola and Emerue}

Haematology and serum indices of wistar rats administered extract from selected phyto-antioxidant sources

The haematology parameters of female Wistar rats administered extract from selected phyto-antioxidant sources are presented in Table 6. There were no significant differences $(\mathrm{P}>0.05)$ in packed cell volume, haemoglobin, $\mathrm{RBC}, \mathrm{WBC}$, lymphocytes, eosinophils, mean corpuscular volume and mean corpuscular haemoglobin across the treatment groups. The serum indices of the female wistar rats administered extract from selected phytoantioxidant sources are presented in Table 7. There were no significant differences $(\mathrm{P}>0.05)$ in all the serum indices examined among the treatments. The haematology parameters and serum indices of male wistar rats administered extract from selected phyto-antioxidant sources are presented in Table 8 and 9. There were no significant differences in all the parameters monitored except for globulin with T7 having higher value of $5.00 \mathrm{~g} / \mathrm{dL}$ than other treatment groups.

\begin{tabular}{|c|c|c|c|c|c|c|c|c|c|}
\hline Parameters & $\mathrm{T} 1$ & $\mathrm{~T} 2$ & $\mathrm{~T} 3$ & $\mathrm{~T} 4$ & $\mathrm{~T} 5$ & T6 & $\mathrm{T} 7$ & T8 & P-value \\
\hline PCV $(\%)$ & $47.00 \pm 3.78$ & $49.00 \pm 0.85$ & $43.00 \pm 5.48$ & $49.50 \pm 1.80$ & $48.17 \pm 1$ & $50.50 \pm 2.99$ & $49.50 \pm 2.01$ & $50.50 \pm 1.82$ & 0.73 \\
\hline $\mathrm{HB}(\mathrm{g} / \mathrm{dL})$ & $15.01 \pm 1.12$ & $15.37 \pm 0.68$ & $13.98 \pm 1.75$ & $16.10 \pm 0.48$ & $15.85 \pm 0.57$ & $16.18 \pm 0.88$ & $16.04 \pm 0.65$ & $16.73 \pm 0.67$ & 0.57 \\
\hline $\mathrm{RBC}\left(\mathrm{x} 10^{6} / \mu \mathrm{L}\right)$ & $7.61 \pm 0.67$ & $7.85 \pm 0.37$ & $7.03 \pm 0.87$ & $8.18 \pm 0.25$ & $7.97 \pm 0.32$ & $8.19 \pm 0.37$ & $8.04 \pm 0.33$ & $8.43 \pm 0.24$ & 0.57 \\
\hline $\mathrm{WBC}\left(\mathrm{x} 10^{3} / \mu \mathrm{L}\right)$ & $5.40 \pm 33.3^{\mathrm{ab}}$ & $5.64 \pm 34.6^{\mathrm{ab}}$ & $4.65 \pm 20.20^{b}$ & $5.39 \pm 1.93^{\mathrm{ab}}$ & $4.65 \pm 2.42^{\mathrm{bc}}$ & $5.97 \pm 3.72^{\mathrm{ab}}$ & $4.89 \pm 67.8^{\mathrm{ab}}$ & $4.89 \pm 5.09^{9 \mathrm{ab}}$ & 0.13 \\
\hline Platelet $\left(\mathrm{x} 10^{3} / \mu \mathrm{L}\right)$ & $130.00 \pm 8.34^{\mathrm{ab}}$ & $115.17 \pm 3.86^{\mathrm{bc}}$ & $138.17 \pm 8.58^{8 \mathrm{ab}}$ & $135.83 \pm 5.23^{\mathrm{ab}}$ & $125.83 \pm 8.56^{\mathrm{b}}$ & $140.17 \pm 4.59^{\mathrm{ab}}$ & $154.60 \pm 1.74^{\mathrm{a}}$ & $106.60 \pm 6.62$ & 0.01 \\
\hline Lymphocyte (\%) & $73.83 \pm 1.33$ & $73.83 \pm 1.66$ & $66.17 \pm 4.00$ & $73.33 \pm 1.67$ & $72.17 \pm 1.40$ & $73.67 \pm 1.58$ & $73.20 \pm 0.66$ & $72.50 \pm 1.15$ & 0.13 \\
\hline Neutrophil (\%) & $23.17 \pm 1.45^{b}$ & $22.67 \pm 1.74^{\mathrm{b}}$ & $30.33 \pm 3.90^{\mathrm{a}}$ & $25.50 \pm 1.38^{\mathrm{ab}}$ & $24.50 \pm 1.57^{\mathrm{a}}$ & $23.50 \pm 2.39^{\mathrm{b}}$ & $23.40 \pm 0.60^{b}$ & $22.67 \pm 1.20^{b}$ & 0.18 \\
\hline Monocyte (\%) & $2.00 \pm 0.26^{\mathrm{ab}}$ & $1.33 \pm 0.21^{\mathrm{b}}$ & $1.50 \pm 0.22^{b}$ & $1.83 \pm 0.17^{\mathrm{ab}}$ & $1.50 \pm 0.22^{b}$ & $1.83 \pm 0.31^{\mathrm{ab}}$ & $1.60 \pm 0.40^{\mathrm{b}}$ & $2.50 \pm 0.34^{\mathrm{a}}$ & 0.09 \\
\hline Eosinophil (\%) & $1.00 \pm 0.26$ & $2.00 \pm 0.52$ & $2.00 \pm 0.52$ & $1.33 \pm 0.56$ & $1.83 \pm 0.54$ & $1.50 \pm 0.56$ & $1.80 \pm 0.37$ & $2.33 \pm 0.21$ & 0.55 \\
\hline $\mathrm{MCV}(\mathrm{fL})$ & $62.28 \pm 1.56$ & $62.31 \pm 1.04$ & $60.89 \pm 1.39$ & $60.53 \pm 0.89$ & $60.49 \pm 0.79$ & $61.45 \pm 1.00$ & $59.93 \pm 0.66$ & $59.92 \pm 1.28$ & 0.67 \\
\hline $\mathrm{MCH}(\mathrm{Pg})$ & $19.94 \pm 0.50$ & $19.61 \pm 0.29$ & $19.78 \pm 0.43$ & $19.70 \pm 0.20$ & $19.91 \pm 0.20$ & $19.72 \pm 0.23$ & $19.95 \pm 0.24$ & $19.85 \pm 0.53$ & 0.99 \\
\hline $\mathrm{MCHC}(\%)$ & $32.03 \pm 0.33^{b c}$ & $31.50 \pm 0.60^{c}$ & $32.50 \pm 0.38^{\mathrm{abc}}$ & $32.57 \pm 0.24^{\mathrm{abc}}$ & $32.92 \pm 0.22^{\mathrm{ab}}$ & $32.10 \pm 0.24^{\mathrm{bc}}$ & $33.29 \pm 0.23^{\mathrm{a}}$ & $33.12 \pm 0.27^{7 \mathrm{~b}}$ & 0.01 \\
\hline
\end{tabular}

${ }^{\mathrm{ab}}=$ means on the same row with different superscripts are significantly $(\mathrm{P}<0.05)$ different.

$\mathrm{PCV}=$ packed cell volume; $\mathrm{HB}=$ haemoglobin; $\mathrm{RBC}=$ red blood cell; $\mathrm{WBC}=$ white blood cell; $\mathrm{Tl}=\mathrm{N} 0$ aflatoxin + No extract (control); T2 $=$ Diet + Aflatoxin + No extract; T3 $=$ Aflatoxin $+100 \mathrm{mg} / \mathrm{kg}$ BW carrot extract; T4 = Aflatoxin $+200 \mathrm{mg} / \mathrm{kg}$ BW carrot extract; T5 = Aflatoxin $+100 \mathrm{mg} / \mathrm{kg}$ BW ginger extract; $\mathrm{T} 6$ $=$ Aflatoxin $+200 \mathrm{mg} / \mathrm{kg} \mathrm{BW}$ ginger extract; $\mathrm{T} 7=$ Aflatoxin $+100 \mathrm{mg} / \mathrm{kg}$ BW garlic extract $; \mathrm{T} 8=$ Aflatoxin

$+200 \mathrm{mg} / \mathrm{kg}$ BW garlic extract.

Table 7: Serum indices of female Wister rats admi nistered extract from selected p hyto-antioxidant sources

\begin{tabular}{llllllllll}
\hline Parameters & T1 & T2 & T3 & T4 & T5 & T6 & T7 & T8 & P-value \\
\hline Total protein $(\mathrm{g} / \mathrm{dL})$ & $6.77 \pm 0.39$ & $7.00 \pm 0.12$ & $7.06 \pm 0.22$ & $6.53 \pm 0.03$ & $7.08 \pm 0.44$ & $6.64 \pm 0.22$ & $7.06 \pm 0.24$ & $6.33 \pm 0.29$ & 0.48 \\
Albumin $(\mathrm{g} / \mathrm{dL})$ & $2.73 \pm 0.12$ & $2.90 \pm 0.15$ & $3.10 \pm 0.12$ & $3.63 \pm 0.12$ & $3.02 \pm 0.22$ & $2.88 \pm 0.17$ & $3.03 \pm 0.14$ & $2.58 \pm 0.08$ & 0.21 \\
Globulin $(\mathrm{g} / \mathrm{dL})$ & $4.03 \pm 0.29$ & $4.10 \pm 0.06$ & $3.97 \pm 0.12$ & $3.90 \pm 0.10$ & $4.06 \pm 0.24$ & $3.76 \pm 0.09$ & $4.03 \pm 0.11$ & $3.75 \pm 0.23$ & 0.79 \\
Arginine ratio & $0.67 \pm 0.03$ & $0.70 \pm 0.06$ & $0.73 \pm 0.03$ & $0.63 \pm 0.07$ & $0.70 \pm 0.03$ & $0.74 \pm 0.04$ & $0.68 \pm 0.03$ & $0.67 \pm 0.04$ & 0.69 \\
AST (uL) & $42.00 \pm 1.15$ & $41.00 \pm 0.58$ & $42.00 \pm 1.33$ & $37.33 \pm 0.33$ & $41.00 \pm 2.49$ & $39.60 \pm 1.54$ & $42.00 \pm 2.12$ & $38.33 \pm 1.52$ & 0.48 \\
ALT(uL) & $30.33 \pm 1.20^{\mathrm{ab}}$ & $29.33 \pm 0.67^{\mathrm{ab}}$ & $32.00 \pm 1.15^{\mathrm{a}}$ & $26.67 \pm 1.20^{\mathrm{b}}$ & $30.00 \pm 1.87^{\mathrm{b}}$ & $28.80 \pm 1.11^{\mathrm{ab}}$ & $30.50 \pm 1.32^{\mathrm{ab}}$ & $28.00 \pm 1.21^{\mathrm{ab}}$ & 0.34 \\
ALP $(\mathrm{uL})$ & $108.00 \pm 12.22$ & $109.00 \pm 11.06$ & $97.33 \pm 12.12$ & $182.00 \pm 98.50$ & $102.80 \pm 9.16$ & $96.80 \pm 5.27$ & $110.25 \pm 4.82$ & $94.00 \pm 5.42$ & 0.46 \\
BUN $(\mathrm{mg} / \mathrm{dL})$ & $16.67 \pm 0.52$ & $16.57 \pm 0.29$ & $17.37 \pm 0.12$ & $16.23 \pm 0.12$ & $17.06 \pm 0.44$ & $16.66 \pm 0.32$ & $17.15 \pm 0.28$ & $16.70 \pm 0.19$ & 0.43 \\
Creatinine $(\mathrm{mg} / \mathrm{dL})$ & $0.57 \pm 0.03$ & $0.60 \pm 0.00$ & $0.63 \pm 0.03$ & $0.53 \pm 0.03$ & $0.60 \pm 0.04$ & $0.58 \pm 0.04$ & $0.58 \pm 0.03$ & $0.53 \pm 0.02$ & 0.47 \\
\hline
\end{tabular}

${ }^{a b}=$ Means on the same row with different superscripts are significantly $(\mathrm{P}<0.05)$ different.

$\mathrm{AST}=$ a spartate amino transferase; $\mathrm{ALT}=$ alanine amino transfe rase; $\mathrm{ALP}=$ alkaline ph osphate; BUN $=$ Blood urea nitrogen; $\mathrm{T} 1=$ No aflatoxin + No extract (control), $\mathrm{T} 2=$ Diet + Aflatoxin + No extract, $\mathrm{T} 3=$ Aflatoxin $+100 \mathrm{mg} / \mathrm{kg} \mathrm{BW}$ carrot extract, $\mathrm{T} 4=$ Aflatoxin $+200 \mathrm{mg} / \mathrm{kg} \mathrm{BW}$ carrot extract, $\mathrm{T} 5=$ Aflatoxin + $100 \mathrm{mg} / \mathrm{kg} \mathrm{BW}$ ginger extract, $\mathrm{T} 6=$ Aflatoxin $+200 \mathrm{mg} / \mathrm{kg} \mathrm{BW}$ ginger extract, $\mathrm{T} 7=$ Aflatoxin $+100 \mathrm{mg} / \mathrm{kg}$ $\mathrm{BW}$ garlic extract, $\mathrm{T} 8=$ Aflatoxin $+200 \mathrm{mg} / \mathrm{kg}$ BW garlic extract. 


\section{Mitigating dietary aflatoxins in Wistar rat using selected phyto-antioxidant sources}

\begin{tabular}{|c|c|c|c|c|c|c|c|c|c|}
\hline Parameters & T1 & $\mathrm{T} 2$ & T3 & T4 & T5 & T6 & $\mathrm{T} 7$ & T8 & P-value \\
\hline $\mathrm{PCV}(\%)$ & $45.00 \pm 1.90$ & $51.25 \pm 2.25$ & $50.00 \pm 1.15$ & $50.25 \pm 1.03$ & $44.50 \pm 10.02$ & $43.75 \pm 5.12$ & $51.25 \pm 3.61$ & $49.60 \pm 2.40$ & 0.79 \\
\hline $\mathrm{HB}(\mathrm{g} / \mathrm{dL})$ & $14.96 \pm 0.56$ & $15.83 \pm 0.49$ & $16.67 \pm 0.35$ & $16.40 \pm 0.31$ & $14.40 \pm 3.36$ & $14.15 \pm 1.81$ & $16.68 \pm 1.29$ & $16.76 \pm 0.82$ & 0.74 \\
\hline $\mathrm{RBC}\left(\mathrm{x} 10^{6} / \mu \mathrm{L}\right)$ & $7.55 \pm 0.34$ & $8.34 \pm 0.29$ & $8.50 \pm 0.16$ & $8.46 \pm 0.09^{\mathrm{a}}$ & $7.23 \pm 1.39$ & $7.23 \pm 0.99$ & $8.38 \pm 0.51$ & $8.27 \pm 0.31$ & 0.66 \\
\hline $\mathrm{WBC}\left(\mathrm{x} 10^{3} / \mu \mathrm{L}\right)$ & $4.41 \pm 5.97$ & $5.88 \pm 1.11$ & $7.61 \pm 3.34$ & $7.59 \pm 2.49$ & $7.15 \pm 7.41$ & $4.58 \pm 6.17$ & $4.09 \pm 1.96$ & $4.29 \pm 1.69$ & 0.27 \\
\hline Platelet $\left(\mathrm{x} 10^{3} / \mu \mathrm{L}\right)$ & $110.18 \pm 5.33^{\mathrm{ab}}$ & $109.57 \pm 2.12^{b}$ & $104.76 \pm 2.00^{\mathrm{a}}$ & $110.72 \pm 1.08^{\mathrm{ab}}$ & $111.20 \pm 1.82^{\mathrm{ab}}$ & $118.70 \pm 1.94^{\mathrm{ab}}$ & $113.95 \pm 2.14^{\mathrm{ab}}$ & $111.92 \pm 4.85^{\mathrm{ab}}$ & 0.43 \\
\hline Lymphocyte (\%) & $71.20 \pm 1.53$ & $69.75 \pm 3.71$ & $73.67 \pm 1.20$ & $72.75 \pm 1.11$ & $69.75 \pm 5.36$ & $71.00 \pm 1.73$ & $74.25 \pm 0.85$ & $74.40 \pm 1.21$ & 0.76 \\
\hline Neutrophil (\%) & $26.00 \pm 1.76$ & $28.00 \pm 3.85$ & $23.67 \pm 1.67$ & $24.50 \pm 0.87$ & $26.50 \pm 4.87$ & $25.75 \pm 1.55$ & $23.00 \pm 1.47$ & $23.00 \pm 1.58$ & 0.81 \\
\hline Monocyte (\%) & $2.20 \pm 0.37$ & $1.50 \pm 0.29$ & $1.67 \pm 0.67$ & $1.00 \pm 0.41$ & $1.25 \pm 0.48$ & $2.00 \pm 0.41$ & $1.50 \pm 0.29$ & $1.20 \pm 0.37$ & 0.40 \\
\hline Eosinophil (\%) & $0.60 \pm 0.40^{b}$ & $0.75 \pm 0.48^{\mathrm{ab}}$ & $1.00 \pm 0.58^{\mathrm{ab}}$ & $1.75 \pm 0.48^{\mathrm{ab}}$ & $2.50 \pm 0.50^{\mathrm{ab}}$ & $1.25 \pm 0.63^{\mathrm{ab}}$ & $1.25 \pm 0.63^{\mathrm{ab}}$ & $1.40 \pm 0.51^{\mathrm{ab}}$ & 0.28 \\
\hline MCV (fL) & $596.27 \pm 3.71$ & $614.18 \pm 7.43$ & $588.15 \pm 3.42$ & $594.38 \pm 11.26$ & $581.98 \pm 34.06$ & $612.67 \pm 18.18$ & $610.76 \pm 14.14$ & $599.16 \pm 10.86$ & 0.79 \\
\hline $\mathrm{MCH}(\mathrm{Pg})$ & $19.84 \pm 0.27$ & $20.19 \pm 0.21$ & $19.60 \pm 0.50$ & $19.40 \pm 0.33$ & $19.06 \pm 1.49$ & $19.68 \pm 0.34$ & $19.84 \pm 0.35$ & $20.24 \pm 0.30$ & 0.85 \\
\hline MCHC (\%) & $33.27 \pm 0.27^{\mathrm{ab}}$ & $32.89 \pm 0.50^{\mathrm{ab}}$ & $33.34 \pm 0.15^{\mathrm{ab}}$ & $32.64 \pm 0.07^{\mathrm{ab}}$ & $32.63 \pm 0.73^{\mathrm{ab}}$ & $32.17 \pm 0.73^{b}$ & $32.51 \pm 0.60^{\mathrm{ab}}$ & $33.79 \pm 0.24^{\mathrm{a}}$ & 0.27 \\
\hline $\begin{array}{l}\mathrm{ab}=\text { means or } \\
\text { PCV = pack } \\
\text { Aflatoxin }+ \\
\text { carrot extrac } \\
\text { extract, T6 = } \\
\text { T8 = Aflatox }\end{array}$ & $\begin{array}{l}\text { n the same row w } \\
\text { ed cell volume, } \\
\text { No extract (contr } \\
\text { t, T4 = Aflatoxin } \\
=\text { Aflatoxin }+20 \\
x i n+200 \mathrm{mg} / \mathrm{kg}\end{array}$ & 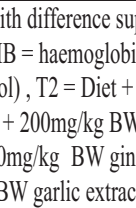 & $\begin{array}{l}\text { erscripts are } \mathrm{s} \\
\mathrm{RBC}=\text { red b } \\
\text { fflatoxin }+\mathrm{No} \\
\text { carrot extract } \\
\text { ex extract, } \mathrm{T} 7\end{array}$ & $\begin{array}{l}\text { gnificantly }(\mathrm{P}<0 \\
\text { ood cell, WBC= } \\
\text { extract, } \mathrm{T} 3=\text { Afl } \\
\text { T5 = Aflatoxin } \\
=\text { Aflatoxin }+10\end{array}$ & $\begin{array}{l}\text { 05) different. } \\
\text { white blood cell, } \\
\text { toxin + } 100 \mathrm{mg} / \mathrm{k} \\
+100 \mathrm{mg} / \mathrm{kg} \mathrm{B}\end{array}$ & $\begin{array}{l}\mathrm{Tl}=\mathrm{N}_{0} \\
\mathrm{BW} \\
\text { ginger }\end{array}$ & & & \\
\hline
\end{tabular}

Table 9: Serum indices of male Wistar rats administered extract from selected phyto -antioxidant sources

\begin{tabular}{|c|c|c|c|c|c|c|c|c|c|}
\hline Parameters & $\mathrm{T} 1$ & $\mathrm{~T} 2$ & $\mathrm{T3}$ & $\mathrm{T} 4$ & T5 & T6 & $\mathrm{T} 7$ & $\mathrm{~T} 8$ & P-value \\
\hline Total protein $(\mathrm{g} / \mathrm{dL})$ & $6.96 \pm 0.05$ & $6.93 \pm 0.41$ & $7.07 \pm 0.12$ & $7.37 \pm 0.31$ & $7.28 \pm 0.69$ & $7.58 \pm 0.40$ & $8.15 \pm 0.24$ & $7.54 \pm 0.30$ & 0.34 \\
\hline Albumine (g/dL) & $2.64 \pm 0.05$ & $5 \pm 0.20$ & $2.90 \pm 0.06$ & $3.15 \pm 0.17$ & $3.10 \pm 0.37$ & $3.08 \pm 0.13$ & $3.15 \pm 0.26$ & $2.80 \pm 0.14$ & 0.46 \\
\hline Globulin (g/dL) & $4.32 \pm 0.09^{b}$ & $4.08 \pm 0.27^{\mathrm{b}}$ & $4.17 \pm 0.09^{b}$ & $4,15 \pm 0.14^{b}$ & $4.18 \pm 0.32^{b}$ & $4.50 \pm 0.27^{\mathrm{ab}}$ & $5.00 \pm 0.07^{\mathrm{a}}$ & $4.74 \pm 0.19^{\mathrm{aab}}$ & 0.04 \\
\hline Arginine ratio & $0.58 \pm 0.02^{b}$ & $0.70 \pm 0.00^{\mathrm{a}}$ & $0.67 \pm 0.03^{\mathrm{ab}}$ & $0.73 \pm 0.03^{\mathrm{a}}$ & $0.70 \pm 0.04^{\mathrm{ab}}$ & $0.65 \pm 0.03^{\mathrm{ab}}$ & $0.65 \pm 0.65^{\mathrm{ab}}$ & $0.56 \pm 0.02^{b}$ & 0.01 \\
\hline AST (UL) & $42.20 \pm 1.39$ & $40.25 \pm 2.50$ & $41.67 \pm 0.08$ & $43.75 \pm 2.36$ & $44.00 \pm 3.03$ & $42.75 \pm 2.29$ & $46.00 \pm 0.58$ & $43.60 \pm 1.78$ & 0.67 \\
\hline $\operatorname{ALT}(\mathrm{uL})$ & $30.60 \pm 1.33^{\mathrm{ab}}$ & $28.75 \pm 1.65^{b}$ & $30.33 \pm 1.20^{\mathrm{ab}}$ & 31.2 & 33.00 & $32.00 \pm 1.68^{\mathrm{ab}}$ & & $32.20 \pm 1.66^{\mathrm{ab}}$ & 0.41 \\
\hline $\operatorname{ALP}(\mathrm{uL})$ & $101.40 \pm 462$ & $115.75 \pm 3.30$ & $111.33 \pm 8.11$ & $115.00 \pm 3.76$ & $109.50 \pm 11.51$ & $110.00 \pm 6.49$ & $112.75 \pm 6.02$ & $113.60 \pm 4.81$ & 0.76 \\
\hline $\mathrm{BUN}(\mathrm{mg} / \mathrm{d}$ & $17.10 \pm 0.11^{\mathrm{ab}}$ & $16.53 \pm 0.43^{b}$ & $16.53 \pm 0.27^{b}$ & $16.90 \pm 0.56^{\mathrm{ab}}$ & $17.60 \pm 11.40^{\mathrm{ab}}$ & $17.60 \pm 0.36^{\mathrm{ab}}$ & $17.95 \pm 0.43^{b}$ & $17.36 \pm 0.41^{\mathrm{ab}}$ & 0.17 \\
\hline Creatinine (mg/dL) & $0.60 \pm 0.00$ & $0.65 \pm 0.04$ & $0.68 \pm 0.00$ & $0.63 \pm 0.05$ & $0.63 \pm 0.05$ & $0.68 \pm 0.03$ & $0.65 \pm 0.03$ & $0.66 \pm 0.02$ & 0.55 \\
\hline
\end{tabular}

$\mathrm{ab}^{\mathrm{b}}=$ Means on the same row with difference superscripts are significantly $(\mathrm{P}<0.05)$ different.

$\mathrm{AST}=$ aspartate aminotransferase, $\mathrm{ALT} \quad=$ alanine amino transferase $\quad \mathrm{ALP}=$ alkaline phosphate, $\mathrm{BUW}=\mathrm{T} 1=$ No aflatoxin + No extract $($ control $), T 2=$ Diet + Aflatoxin + No extract, $\mathrm{T} 3=$ Aflatoxin $+100 \mathrm{mg} / \mathrm{kgBW}$ carrot extract, $\mathrm{T} 4=$ Aflatoxin $+200 \mathrm{mg} / \mathrm{kg} \mathrm{BW}$ carrot extract, $\mathrm{T} 5=$ Aflatoxin + $100 \mathrm{mg} / \mathrm{kg}$ BW ginger extract, $\mathrm{T} 6=$ Aflatoxin $+200 \mathrm{mg} / \mathrm{kg}$ BW ginger extract, $\mathrm{T} 7=$ Aflatoxin $+100 \mathrm{mg} / \mathrm{kg}$ $\mathrm{BW}$ garlic extract, $\mathrm{T} 8=$ Aflatoxin $+200 \mathrm{mg} / \mathrm{kg}$ BW garlic extract

\section{Discussion}

The Wistar male and female rats exposed to 200mg Garlic extract had the best feed conversion ratio and weight gain than other treatment groups which suggest that the garlic extract had significant positive effect on the growth performance of the Wister rats better than both the positive and the negative control groups. It implies that phyto-antioxidant in garlic help inducing growth rate than other treatment groups. This agrees with the report of Rahman et al. (2011) that ginger flavonoids contained anti-oxidant activity which may probably reduce stress in the aflatoxin contaminated wistar rats. It has been reported previously that feed intake was not affected (Onimisi et al., 2007), feed conversion ratio was reduced (Moorthy et al., 2009), while water consumption was stimulated by the inclusion of ginger in the diet of broiler chickens (Onimisi et al., 2007). Several phytochemicals have been tested in early stages and have been shown to have anabolic effect (Devi et al., 2015; González-Ríos et al., 2016). These findings agreed with the report of Halliwell (1994) that the consumption of phyto-chemical 


\section{Ewuola and Emerue}

rich foods such as fruits, vegetables, spice are associated with a reduced risk of diseases mediated by oxidative stress and inflammation such as certain cancers, atherosclerosis and neurodegenerative diseases. It also corroborated the report of Jiang et al. (2007) and Li et al. (2015) who reported positive results on the growth performance of animal feed supplemented with phyto-chemicals. Non-significant effect of the extracts on eviscerated and organs weight implies that they were not adversely affected. Research has shown that several phytochemicals have been previously reported to have an anabolic activity (Jiang et al., 2007; Herrera et al., 2011; Macías-Cruz 2014; Devi et al., 2015; González-Ríos et al., 2016), garlic improved meat and carcass quality (Kim et al., 2009). Phytochemicals in other spices such as ginger rhizome have been reported to stimulate growth performance (Ademola et al., 2009), and in biological systems, $\beta$ carotene functions as a free radical-trapping agent and single oxygen quencher and have anti-mutagenic, chemo-preventive, photoprotective and immune-enhancing properties (Deshpande et al., 1995). Serum indices of Wistar rats administered with selected Phyto-antioxidant extract that was not significantly affected among the treatments possibly affirm that the three extracts did not adversely affect the organ functions and serum variables. According to Keller et al. (2005) contamination of medicinal plants with mycotoxins can contribute to adverse human health problems and therefore represents a special hazard. Low levels of aflatoxin exposure require continuous consumption for several weeks to months in order for signs of liver dysfunction to appear (Bingham et al., 2003). In the past decade carotenoids such as $\beta$-carotene have attracted considerable attention because of their possible protective effect against some types of cancers (Bast et al., 1996; Santo et al., 1996; Van, 1996), Ginger, the rhizome of the Zingiber officinale, plays an important role in prevention of diseases. But the exact mechanism of action in diseases management is not fully understood. It is thought that the extract act as anticancer due to various constituents such as vallinoids, viz. -gingerol and -paradol, shogaols, zingerone, and galanals $\mathrm{A}$ and $\mathrm{B}$ in ginger (Aggarwal, and Shishodia, 2006) and also Allicin has been found as the major biologically active component of garlic as reported by Cavallito and Bailey (1994), allicin is the key ingredient responsible for the broad- spectrum of anti-bacterial activity in garlic. The haematological parameters examination is among the methods which may contribute to the detection of some changes in health and physiological status, which may not be apparent during physical examination but which affects the fitness of the animal (Bamishaiye et al., 2009). In this present study it was found that the haematological parameters of the female wistar rat studied revealed no significant differences in the values obtained which suggests that the aflatoxin did not have any deleterious effect on the parameters when mitigated with phyto-antioxidant. There was no significant difference found in the haematological indices of the male and female wistar rats administered extract from selected phytoantioxidant sources, which suggests that the aflatoxin contaminated diet did not have any abysmal effect on the haematological parameters of the wistar rats examined when mitigated with any of the phytoantioxidants used in this study, which corroborate the report of (Chineke et al., 2006) which stated that asides from genotype, age, sex, differences in haematological parameter may be attributed to nutritional, environmental and hormonal factors. 
Mitigating dietary aflatoxins in Wistar rat using selected phyto-antioxidant sources

\section{Conclusion}

The result of this study has shown that the phyto-chemical present in th plant extract administered to the wistar rats mitigated the adverse effect of the aflatoxin contaminated feed and improved the growth of the wistar rats. Wistar rats that were administered selective phyto-antioxidant plant extracts at different graded levels were found to perform better than the control groups. Therefore, the selected phyto-antioxidants especially $200 \mathrm{mg} / \mathrm{kg}$ body weight of garlic extract could be used to mitigate aflatoxin effect in livestock feed without any deleterious effects on haematological parameters.

\section{Recommendations}

The selected phyto-chemicals especially $200 \mathrm{mg} / \mathrm{kg}$ body weight garlic extract should be used to mitigate aflatoxin effect in livestock feed without any deleterious effects on performance, carcass yield, haematological parameters and the consumers. Higher graded level of concentration of the extract should also be further researched into.

\section{Conflict of interest}

Authors declared that no conflict of interest exist concerning this manuscript.

\section{References}

Ademola, S. G., Farinu, G. O. and Babatunde, G. M. 2009. Serum lipid, growth and haematological parameters for broilers fed garlic, ginger and their mixtures. World Journal of Agricultural Sciences 5, 99-104

Adugna, S., Alemu, L. A. M., Kelemu, T., Tekola, H., Kibret, B. and Genet, S. 2004. Medical Biochemistry: Lecture notes for health science students. Ethopia public health training initiative Pp. 1-19

Aggarwal, B. B. 2014. Molecular targets of dietary agents for prevention and therapy of cancer. Biochem Pharmacol; 71:1397-1421.

Al-Yahya, M. A., Rafatullah, S., Mossa, J. S., Ageel, A. M., Parmar, N. S., Tariq, M. 1989. Gastroprotective activity of gingerzingiber officinal rocs, in albino rats. Am J Chin Med.; 17:51-56.

Ammon, H. P. T., Safayhi, H., Mack, T., and Sabieraj, J. 1993. Mechanism of anti- inflammatory actions of curcumine and boswellic acid. Journal of Ethnopharmacology 38, 105-112.

Anon 1952. The wealth of India: raw materials, vol 3. Council of Scientific and Industrial Research, New Delhi, Pp 20-21

Antony, S. R., and Kuttan, G. 1999. Immunomodulatory activity of curcumin. Immunol Invest., 28: 291-303.

Bast, A, Van den Berg, H, Van der Plas, $R$. M, Haenen G. R. M. 1996. $\beta$ Carotene as antioxidant. Eur J Clin Nutr 50:554-556.

Bamishaiye, E. Muhammad, N. and B a m is h a i ye, O. ( 2009$)$. Haematological parameters of albino rats fed on tiger nuts (Cyperus esculentus) tuber oil meat based diet. The internal Journal of Nutrition and wellness, 10(1): 1857 -1881 .

Bingham, A. K., Phillips, T. D., Bauer, J. E. 2003. "Potential for dietary protection against the effects of aflatoxins in animals". Journal of the American Veterinary Medical Association. 222 (5): 591-600.

Cavalito, C., Small, L. V. and Bayley, J. H. 1947. Alkyl Thiosulphinates $J$. Am. Chem. Soc, 69: 1710-1713.

Charoenpornsook, K. and Kavisarasai, K. 2006. Mycotoxin in animal feed stuff of Thailand. KMILT Sci. Tech.J., 6:25-28. 
Chineke, C. A, Ologun, A. G. and Ikeobi, C. O. N. 2006. Haematological parameters in rabbit breeds and crosses in humid topics, Pakistan Journal of Biological Sciences, 9:2102-2106.

Chowdhury, S. R, Chowdhury, S. D, Smith, T. K 2002. Effect of dietary garlic on cholesterol metabolism in laying hen. Journal of Poultry Science 7(2): 122-128.

Cortyl, M. 2008. Mycotoxins in animal nutrition-problems and solutions. Mycotoxins animal feed science technol., 69: 155-166.

D'odorico, A., Martines, D., Naccarato, R., and Baraglota, A. 2000. Plasma antioxidant levels in chronic cholestatic liver diseases. Allimentary Pharmacology and Therapeutics, 14(3): 353-358.

Deshpande, S. S., Madhavi, D. L and Salunkh, D. K. 1995. Food Antioxidants: Technological. Toxicological and Health perspectives. CRC. Press agris.fao.ng. Technology and Engineering/Food Science pp 473.

Devegowda, G. M. V. L., Radu, A., Nazar and H. V. L. M. Swany, 1998. Mycotoxin picture worldwide: Novel Solutions for their counteraction. Proceedings of Alltech's $14^{\text {th }}$ Annual Symposium on Biotechnology in feed industry. Passport of the year 2000, (BFI'98), Nottingham University press, pp: 241-255

Devi, S. M, Park, J. W, Kim, I. H. 2015. Effect of plant extracts on growth performance and insulin-like growth factor 1 secretion in growing pigs. Rev Bras Zootec. 44:355-360.

Ewuola, E. O and Egbunike, G. N. 2008. Haematological and serum biochemical response of growing rabbits buck fed different levels of dietary Fumonism $\mathrm{B}_{1}$. African Journal of Biotechnology, 7(23): 4304-4309.

Eric, B. 2010. "Garlic and other Allinins: The Lore and the Science "Cambridge, Royal Society of Chemistry 34: 152166.

Girling, S. J., Campbell-Palmer, R., Pizzi, R., Fraser, M. A., Cracknell, J. and Arnemo, J. 2015. Haematology and Serum Biochemistry Parameters and Variations in the Eurasian Beaver (Castor fiber). Contemp Top Lab Anim Sci. 1997; 36:97-99.

Gomez-Espinosa, D., CervantesAnguilar, F. J.; Del Rio- Garcia, J. C; Villarreal-Barajas, T.; Vazquez-Duran, A.; MendezAlbores, A. 2017. Ameliorative Effects of Neutral Electrolyzed water on Growth performance, Biochemical constituents, and histopathological changes in Turkey poults during Aflatoxicosis. Toxins, $9 ; 104$

González-Ríos, H., Dávila-Ramírez, J. L., Peña-Ramos, E. A., Valenzuela-Melendres, M., Zamorano-García, L., IslavaLagarda, T. Y., ValenzuelaGrijalva, N. V. 2016. Dietary supplementation of ferulic acid to steers under commercial feedlot feeding conditions improves meat quality and shelf life. Anim Feed Sci Tech.; 222:111-121.

Halliwell, B. 1994. Oxygen - derived species; their relation to human disease and environmental stress. Environmental health perspective J. Neurochem, 59(5): 1609-1623.

Herrera, R., Alejo, M. L., Asaff A. J. 2011. Methods to accelerate muscle development, decrease fat deposits, and enhance feeding efficiency in 
Mitigating dietary aflatoxins in Wistar rat using selected phyto-antioxidant sources

pigs. Journal of Animal Science and biotechnology. 92: 199-28

Horton, G. M. J., Blethen, D. B. and Prasad, B. M. 1991. The effect of garlic (Allium sativum) on feed palatability of horses and feed consumption selected performance and blood parameters in sheep and swine. Canadian Journal of Animal Science 71: 122-128.

Ito, Y., Peterson S. W. and Wicklow, T. D. 2001. Aspergillus pseudotamarii, a new aflatoxin producing species in Aspergillus Section flavi. Mycol. Res., 105: 233-239.

Jang, I. S., Ko, Y. H., Kang, S. Y., and Lee, C. Y. 2007. Effect of a commercial essential oil on growth performance, digestive enzyme activity and intestinal microflora population in broiler chickens. Animal feed Science and Technology 134, 304-315

Keller, N. P., Turner, G., Bennett, J. W. 2005. "Fungal secondary metabolism-from biochemistry togenomics" .Nat.Rev.Microbiol. 3 (12):93747.

Kim, Y. J. and Yang, H. S. 2009. Effect of dietary garlic bulb and husk on the physiochemical properties of chicken meat. Poultry Science 88, 398-405.

Kohn, R. A. and Allen, M. S. 1995. Enrichment of proteolytic activity relative to nitrogen in preparation from the rumen for in vitro studies. Anim. Feed Sci. and technol. 52: 114.

Kurtzman, C. P., Horn, B. W. and Hesseltine, C. W. 1987. Aspergillus nominus, a new aflatoxin producing species related to Aspergillus flavus and Aspergillus Tamarii. Antonie Van Leeuwenhork, 53: 147-158.

Li, Y. J., Li, L. Y., Li, J. L., Zhang, L.,
Gao, F. and Zhou, G. H. 2015. Effects of dietary supplementation with ferulic acid or vitamin $E$ individually or in combination on meat quality and antioxidant capacity of finishing Pigs. AsianAustral J Anim Sci.; 28:374-381.

Macías-Cruz, U., Perard, S., Vicente, R., Álvarez, F. D., TorrenteraOlivera, N. G., González-Ríos, H., Soto-Navarro, S. A., Rojo, R., Meza-Herrera, C. A. and Avendaño-Reyes, L. 2014. Effects of free ferulic acid on productive performance, blood metabolites, and carcass characteristics of feedlot finishing ewe lambs. J Anim Sci.;92:5762-5768.

Mahima, A., Rahal, R., Deb, S. K., Latheef, F. S. and Somal H. A. 2012. Immunomodulatory and therapeutic potential herbal, traditional/indigenous and ehtinoveterinary medicines. Pak. $J$. Biol. Sci, 15: 754- 774.

Mahima, A. K., Verma, R., Tiwan, K., Karthik, S., Chakraborty, R. D. and D h a m a, K. 2013 . Nutraceuticals from fruits and vegetables at a glance. A review $J$. Biol. Sci, 13:28-47.

McComb, R. B., Bowers, G. N. and Rosen, S. 1998. Alkaline phosphate. Plenum Press, New York. Pp 78-86.

Mohan, R. M., Sargar, B. M. and Anuse, M. A. 2004. Highly Selective Liquid- Liquid extraction of niobium (V) from halide media with high molecular weight amines. Revue Roumaine de Chimie 49(6). 531-537

Moorthy, M., Ravi, S., Ravikuma, M., Viswanathan, K. and Edwin, S. C. 2007. Ginger, Pepper and curry leaf powder as feed additives in broiler diet. Int. J. Poult. Sci. 8: 
779-782.

Moyo, B., Masika, P. J., Hugo, A. and Muchenje, V. 2011. Nutritional characterization of Moringa (Moringa oleifera Lam) Leaves. African Journal of biotechnology 10(60): 12925-12933.

Onimisi, P. A., Dafwang, I. I. and Omage, J. J. 2007. Growth performance and water consumption pattern of broiler chicks fed graded levels of ginger waste meal. Journal of Agriculture, forestry and the Social Sciences 3, 113-199.

Payne, G. A. 1998. Process of contamination by aflatoxin producing fungi and their impacts on crops. In: Mycotoxin in Agriculture and food safety, New York, pp 279-306

Rehman, S. U., Durran, F. R., Chand, N., Khan, R. U and Rehman, F. U. 2011. Comparative efficacy of different schedules of administration of medicinal plants infusion on hematology and serum biochemistry of broiler chicks. Res.opin. Anim.vet. Sci, 1:8-14.

Rodwell, V. W. and Kennelly, J. P. 2013. Enzymes: Mechanism of action In: Harpers illustrated biochemistry ED: Lange Medical Books McGraw-Hill co. New York. Pp, 58.

Soni, K. B., Lahiri, M., Bhide, S. V. and Kuttan, R. 1997. Protective effect of food additives on aflatoxininduced mutagenicity and hepatocarcinogenicity. Cancer Letters, 155: 129-33.

Taylor, G. T. 1975. Male aggression in the presence of an estrous female. Journal of Comparative and Physiological Psychology 89(3):246-52.
Tollba, A. A. H. and Hassan, M. S. H. 2013. Using some natural additives to improve physiological and productive performance of broiler chicks under high temperature conditions. Black cumin (Nigella sativa) or garlic (Allium sativum). Poult. Sci., 23:327-340.

Toro, G. and Ackermann, P. G. 1975. Practical clinical chemistry. Little, Brown and Company. Boston.

Van, P. G. 1996. Review: epidemiological evidence for $\beta$-carotene in prevention of cancer and cardiovascular disease. Eur J Clin Nutr 50:557-561

Wilson, N. M., McMaster, N., Gantulga, D., Soyars, C., McCormick, S. P., Knott, K., Senger, R. S and Sch m a le, D. G. 2017 . Modification of Mycotoxin Deoxynivalenol Using Microorganism Isolated from Environmental Samples. $J$. Toxicology, 9, 141.

Zhang, G. F., Yang, Z. B., Wang, Y., W. R., Jiang, S. Z and Gai, G. S. 2009. Effects of ginger root (Zingiber officinale) processed to different particle sizes on growth performance, antioxidant status, and serum metabolites of broiler chickens. Poultry Science, 88: 2159-2166.

Received: $14^{\text {th }}$ October, 2020 Accepted: $5^{\text {th }}$ February, 2021 